\title{
Object-Based Morphological Profiles for Classification of Remote Sensing Imagery
}

\author{
Christian Geiß, Member, IEEE, Martin Klotz, Andreas Schmitt, Hannes Taubenböck \\ German Aerospace Center (DLR), German Remote Sensing Data Center (DFD), Münchner \\ Straße 20, 82234 Oberpfaffenhofen-Weßling, Germany \\ tel.: +49815328 1255, fax: +498153281445 \\ e-mail: christian.geiss@dlr.de; martin.klotz@dlr.de; andreas.schmitt@dlr.de; \\ hannes.taubenboeck@dlr.de
}

ABSTRACT - Morphological operators (MOs) and their enhancements such as morphological profiles (MPs) are subject to a lively scientific contemplation since they are found to be beneficial for e.g., classification of very high spatial resolution panchromatic, multi- and hyperspectral imagery. They account for spatial structures with differing magnitudes and, thus, provide a comprehensive multi-level description of an image. In this paper, we introduce the concept of object-based morphological profiles (OMPs) to also encode shape-related, topological, and hierarchical properties of image objects in an exhaustive way. Thereby, we seek to benefit from the so-called objectbased image analysis framework by partitioning the original image into objects with a segmentation algorithm on multiple scales. The obtained spatial entities (i.e., objects) are used to aggregate multiple sequences obtained with MOs according to statistical measures of central tendency. This strategy is followed to simultaneously preserve and characterize shape properties of objects and enable both the topological and hierarchical decomposition of an image with respect to the progressive application of MOs. Subsequently, supervised classification models are learned considering this additionally encoded information. Experimental results are obtained with a random forest classifier with heuristically tuned hyperparameters and a wrapper-based feature selection scheme. We evaluated the results for two test sites of panchromatic WorldView-II imagery, which was acquired over an urban environment. In this setting, the proposed OMPs allow for significant improvements with respect to classification accuracy compared to standard MPs (i.e., obtained by paired sequences of erosion, dilation, opening, closing, opening by top-hat, and closing by top-hat operations).

InDEX TERMS: Very High Resolution Imagery, Supervised Classification, LULC Classification, Mathematical Morphology, Morphological Profiles, Object-based Image Analysis 


\section{INTRODUCTION}

The development of methods for the derivation of thematic information such as land use / land cover (LULC) classes from remote sensing imagery has been a major research subject of the remote sensing community in the past decades. Thereby, varying ground sampling distances of individual sensors induced the development of diverse methodological approaches. In this paper, we focus on situations where the ground sampling distance is much smaller than the objects of interest of a scene. This situation can occur in various remote sensing data, depending on the relation of ground sampling distance and corresponding size of the objects of interest [1]. Nowadays, especially data from sensors with a very high spatial resolution such as WorldView I-III, or GeoEye, among others, allow for detailed LULC mapping. At the same time, the high spatial resolution can induce high intra-class and low inter-class variability in particular in heterogeneous environments such as urban areas. This can decrease accuracy of the classification model and induce the well-known salt and pepper effect [1]. One of the most prominent ways to cope with this problem and impose coherent spatial regularization is to compute features which account for the neighborhood of an individual pixel. Among them, features that can be attributed to the family of mathematical morphology [2] allowed for a significant increase of classification accuracy compared to results obtained with the exclusive use of spectral signatures of individual pixels [3].

Nowadays, the application of mathematical morphology [4] is still under a vivid scientific contemplation. From the early 2000s, numerous variations and extension of morphological operators (MOs) were postulated for remote sensing data processing. Pesaresi and Benediktsson [5] introduced an approach based on differential morphological profiles (DMPs) for segmentation of very high resolution imagery. In subsequent works, Benediktsson et al., [6], [7] deployed DMPs for classification of panchromatic and hyperspectral imagery, respectively. Generally, morphological profiles (MPs) allow to compile a comprehensive feature set, which is constituted by a sequential application of geodesic opening and closing (i.e., obtained with opening and closing by reconstruction operators) with varying sizes of the structuring element (SE) to model multi-level structural information of an image. Fauvel et al., [8] complemented this approach by considering the full spectral information for classification. Additionally, in Fauvel et al. [9], [10] a so-called morphological neighborhood system (implemented as a set of connected pixels with an identical gray value), was designed by using morphological area filtering to consider the spectral information surrounding an individual image element (i.e., a pixel). There, also a tailored classification approach was deployed by relying on a Support Vector Machines classifier with individually learned spatial and spectral kernels. Besides, a number of problems related to the sequential application of MOs were addressed. In this sense, Huang et al., [11] investigate several strategies for establishing the base images for further morphological processing. Moreover, Daamouche et al., [12] propose an optimization approach to automatically tailor both the shape and size of the SE with respect to the classification task, and Lv et al., [13] consider differently shaped structuring elements for classification.

However, recently, Dalla Mura et al., [14] introduced a generalization of MPs to the remote sensing community termed morphological attribute profile (AP). Concordant with the aforementioned formulations, APs build upon operators of geodesic reconstruction and provide a multi-level characterization based on sequences of morphological attribute filters 
(AFs) [15]. AFs are morphologically connected filters that allow for processing an image by merging existing flat regions. Thereby, an image is decomposed by iteratively thresholding the connected components. This enables computation of additional features, which characterize the obtained discrete regions such as shape-related measures. Those features were shown to be able to enhance classification accuracy [14]. Yet, APs are very popular and were already applied for classification of hyperspectral images [16], [17] and change detection [18]. Also the concept of sparsity was deployed within this framework for segmentation [19] and classification [20] purposes. A recent review devoted to the application of APs for remote sensing data processing is provided by Ghamisi et al., [21].

In parallel, since the classic paper from Kettig and Landgrebe [22], a huge body of scientific literature arose that deals with the processing of aggregated image elements (i.e., objects) for classification. This subject is referred to as object-based image analysis (OBIA) [23]. One of the primal constituting aspects of such techniques is to model meaningful realworld objects before further processing. Those allow for a diversified characterization of spectral values (i.e., the use of e.g., mean, median, minimum, or maximum spectral values of objects compared to the singular spectral values of pixels), consideration of geometry-related properties of objects, and also encoding of additional spatial information such as relationships of (topological) neighborhood and spatial hierarchy [23]. In this sense, comprehensive multilevel classification approaches, which rely on core OBIA techniques can be found in e.g., [24]-[26]. The past and current popularity of the affiliated conceptual and methodological canon inspired researchers already to categorize it as a paradigm in the context of remote sensing and geographic information science [1] according to Kuhn's theory on the structure of scientific revolutions [27].

In this paper, we seek to combine MOs and OBIA techniques and internalize both processing principles. Therefore, we introduce the concept of object-based morphological profiles (OMPs). In parallel to the sequential application of MOs with varying size of the SE, the non-transformed image is subject to segmentation at multiple levels (i.e., scales). Subsequently, the transformed image information (i.e., obtained by the sequential application of MOs) is aggregated with respect to the generated image objects. For this purpose, we evaluate the applicability of different statistical measures of central tendency (i.e., mean, mode, median). This procedure is designed to avoid shape-related noise frequently induced by the SE. Moreover, it is not limited to the use of openings and closings by reconstruction regarding the underlying MOs to preserve the shapes of objects in the image. In addition, one can obtain information related to the gray-level characteristics and assemblage of discrete regions (i.e., the actual image objects). Overall, it is intended to allow for the computation of discriminative features in a very flexible way: Features can be derived, which describe the shape characteristics of the modelled objects on multiple spatial levels. Simultaneously, hyperparameters of the segmentation method allow controlling preferred shape properties. Moreover, in contrast to previous approaches described above, we consider gray-level characteristics of transformed image information of adjacent discrete regions (beyond the gray-level information surrounding an individual image element (pixel)). To demonstrate the relevance of OMPs, we compare them to classification results obtained with conventional sequentially applied MOs (i.e., sequences of erosion, dilation, opening, closing, opening by top-hat, and closing by top-hat operations) on panchromatic (i.e., single band) imagery. 
The remainder of the paper is organized as follows. We reveal important morphological operators and the concept of MPs in section II. In section III, we introduce the concept of OMPs and provide a formal definition. Experimental data and setup is presented in section IV and actual results of experiments are reported in section V. Concluding remarks are given in section VI.

\section{MORPHOLOGICAL OPERATORS AND PROFILES}

MOs are a family of filters based on set theory [2], [28]. They are based on the execution of minimum and maximum filters with a SE $B_{k}$, e.g., a square window of size $k \times k$, on an image $I$. In the terminology of mathematical morphology, minimum filtering represents an erosion operation $\varepsilon$ extended to grayscale images. It is defined as the minimum of the translations of $\boldsymbol{I}$ by vectors $-\mathbf{b}$ of $B$ [2, p. $66 \mathrm{ff}$.$] :$

$$
\varepsilon_{B}(\boldsymbol{I})=\bigwedge_{\mathbf{b} \in B} \boldsymbol{I}_{-\mathbf{b}}
$$

Analogously, maximum filtering, which represents a dilation operation $\delta$ is defined as follows:

$$
\delta_{B}(\boldsymbol{I})=\bigvee_{\mathbf{b} \in B} \boldsymbol{I}_{-\mathbf{b}} \text {. }
$$

An opening is obtained by the sequential application of a dilation operation to the result of an erosion operation:

$$
\gamma_{B}(\boldsymbol{I})=\delta_{B} \circ \varepsilon_{B}(\boldsymbol{I})
$$

Consequently, a closing is obtained by the sequential application of an erosion to the result of an dilation operation:

$$
\phi_{B}(\boldsymbol{I})=\varepsilon_{B} \circ \delta_{B}(\boldsymbol{I}) .
$$

It can be noted that although opening and closing are combinations of dilation and erosion, they are idempotent. That is, $\gamma_{B}(I)$ and $\phi_{B}(I)$ are not affected by reapplying the opening and closing operator, respectively [29]. To complement these operators, so-called top-hat transforms can be considered. They represent the residuals of an opening or closing, respectively, when compared to the original image. A white top-hat transform shows the bright peaks of $\boldsymbol{I}$ and is obtained with respect to an opening:

$$
T_{w_{B}}(\boldsymbol{I})=\boldsymbol{I}-\gamma_{B}(\boldsymbol{I}) \text {. }
$$

Analogously, a black top-hat transform shows the dark peaks (valleys) of $\boldsymbol{I}$ and is obtained with respect to a closing:

$$
T_{b_{B}}(\boldsymbol{I})=\phi_{B}(\boldsymbol{I})-\boldsymbol{I} .
$$

Generally, the sequential application of MOs with varying size of $B$ allows for the computation of MPs. In literature, one can find some ambiguous definitions with respect to a MP. Authors such as Benediktsson et al., [6], [7] Fauvel et al., [8], or Dalla Mura et al., [14], only refer to an MP, when geodesic opening and closing operations (i.e., opening and closing by reconstruction) are considered. In contrast, Daamouche et al., [12] or Hou et al., [30] follow a less restrictive definition and use the term MP also when simple opening and closing 
operations are considered. However, here, we use the term to describe the concatenation of completing MOs, such as erosion and dilation, opening and closing, or white top-hat transform and black top-hat transform applied in a sequential manner to $\boldsymbol{I}$ with varying size of $B$. They are denoted with $\alpha_{B_{k}}(\boldsymbol{I})$ and $\beta_{B_{k}}(\boldsymbol{I})$. As such, the first part of the MP is defined as follows:

$$
A P_{k}(\boldsymbol{I})=\alpha_{B_{k}}(\boldsymbol{I}), \forall k \in[0, n]
$$

where $\alpha_{B_{k}}$ is the first operation with a SE of size $k$ from 0 to $n$. Consequently, the second part of the MP is defined as follows:

$$
B P_{k}(I)=\beta_{B_{k}}(I), \forall k \in[0, n]
$$

where $\beta_{B_{k}}$ is the second operation with a SE of size $k$ from 0 to $n$. The actual MP is obtained by collation of both sequences:

$$
M P(I)=\left[B P_{n}(\boldsymbol{I}), B P_{n-1}(\boldsymbol{I}), \ldots, B P_{1}(\boldsymbol{I}), \boldsymbol{I}, A P_{1}(\boldsymbol{I}), \ldots, A P_{n-1}(\boldsymbol{I}), A P_{n}(\boldsymbol{I})\right]
$$

This way, a feature vector with a dimensionality of $2 n+1$ is created. Naturally, $A P_{0}(I)$ and $B P_{0}(I)$ correspond to $I$, which is located in the center of the profile.

\section{Aggregated Morphological Operators and Object-Based Profiles}

An overview of the approach for computation of OMPs and subsequent processing is given in Fig. 1.

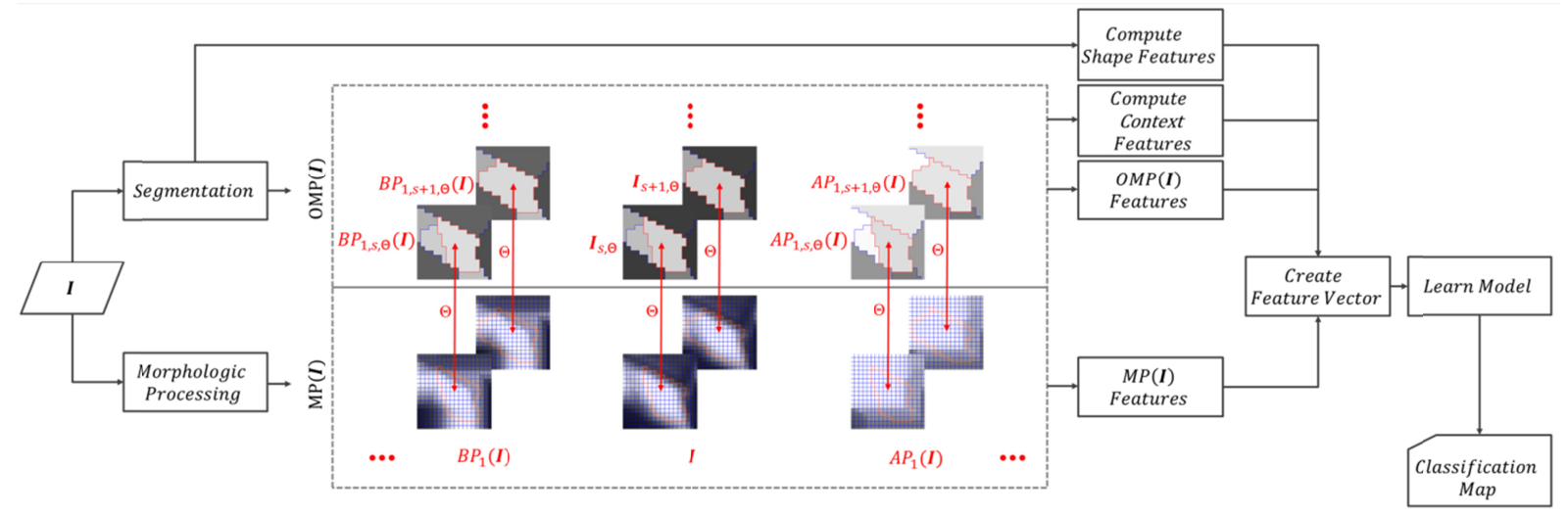

Fig. 1. Overview of the OMP approach. The original image $\boldsymbol{I}$ is processed in two ways. First, conventional morphological profiles $M P(\boldsymbol{I})$ are obtained from $\boldsymbol{I}$. In addition, $\boldsymbol{I}$ is subject to a multi-level segmentation procedure. The obtained image objects are used to aggregate $M P(I)$ with an aggregation function $\Theta$. The corresponding features $(O M P(I))$ can be employed in conjunction with spatial context and shape-related features describing the individual image objects and their spatial context. Finally, the created feature vector is used for learning a supervised classification model.

In parallel to the computation of conventional MPs, $\boldsymbol{I}$ is subject to a segmentation procedure to model image objects. Since it may often be necessary to account for objects in the image with various sizes, a multi-level, hierarchical segmentation procedure can be adopted [22]. I is partitioned with an arbitrary segmentation algorithm at a generic segmentation level $s$ in $N^{s}$ objects $O_{l}^{S}\left(l=1,2, \ldots, N^{S}\right)$. If more than a single segmentation level is considered, an unambiguous hierarchy of levels can be established by implementing the following constraint: 


$$
\bigcup_{O_{l}^{S} \subseteq O_{j}^{s+1}} O_{l}^{s}=O_{j}^{s+1} .
$$

This relation ensures that an object at segmentation level $s$ must be included in only one object at level $s+1$ [24] and thus allows building a consistent profile. Thereby, a defined number of consecutive segmentations $m$ can be carried out to establish an exhaustive set of hierarchical image levels $\Psi \in\left[s, s+1, \ldots, s_{m}\right], s_{i}<s_{i+1}$. Then, a new grey-level value is assigned to an object $O$ based on the grey-level value of corresponding pixels using an arbitrary aggregation function, which is denoted with $\Theta$. In this paper, we deploy three statistical measures of central tendency, namely, mean $(\bar{x})$, mode $(\mathrm{Mo})$, and median $(\tilde{x})$. It can be noted that e.g., statistical measures of spread (variance, or standard deviation) can be considered less relevant in this context since morphological operators impose prior homogeneity constrains on the transformed image.

In concordance with the definition of a MP, we consider the first part of an OMP as follows:

$$
A P_{k, \Psi, \Theta}(\boldsymbol{I})=\Psi_{\psi}(\boldsymbol{I}) \Theta \alpha_{B_{k}}(\boldsymbol{I}), \forall k \in[0, n]
$$

where $\alpha_{B_{k}}$ is the first operation with a SE of size $k$ from 0 to $n, \Psi_{\psi}(I)$ represents the set of hierarchical segmentations obtained from the non-transformed image $\boldsymbol{I}$, and $\Theta$ is the aggregation function. Analogously, the second part of the OMP is defined as follows:

$$
B P_{k, \Psi, \Theta}(\boldsymbol{I})=\Psi_{\psi}(\boldsymbol{I}) \Theta \beta_{B_{k}}(\boldsymbol{I}), \forall k \in[0, n]
$$

where $\beta_{B_{k}}$ is the second operation with a SE of size $k$ from 0 to $n$. The actual OMP is obtained by collation of both sequences:

$O M P(I)$

$=\left[B P_{n, \Psi, \Theta}(\boldsymbol{I}), B P_{n-1, \Psi, \Theta}(\boldsymbol{I}), \ldots, B P_{1, \Psi, \Theta}(\boldsymbol{I}), \boldsymbol{I}_{\Psi, \Theta}, A P_{1, \Psi, \Theta}(\boldsymbol{I}), \ldots, A P_{n-1, \Psi, \Theta}(\boldsymbol{I}), A P_{n, \Psi, \Theta}(\boldsymbol{I})\right.$

Consequently, the feature vector of an OMP corresponds to a dimensionality of $(2 n+1) * m$. An OMP based on a single segmentation level is exemplified in Appendix A [Fig. 6]. In this example $\alpha_{B}(\boldsymbol{I})$ represents a closing and $\beta_{B}(\boldsymbol{I})$ an opening.

A favorable property of OMPs and their inherent processing techniques is the ability to derive further features based on generated discrete image regions (i.e., objects). In this paper, we consider two groups of features. The first group comprises shape-related features, whereas the second group contains contextual features describing (topological) neighborhood relationships. The shape-related features are retrieved to account for distinctively diverse geometric properties of LULC objects. For instance, in urban environments natural objects such as vegetation feature frequently a non-rectangular shape, whereas man-made objects such as buildings feature rectangular shapes, which can be employed for learning a discriminative classification model. A comprehensive number of measures can be found in literature to encode such relations. In this manuscript, we characterize the extent of modelled objects by computing area and perimeter. In addition, widely deployed measures that provide an approximate comparison of an object's shape with 2-D geometrical forms such as rectangle, circle or ellipse are included. Here, five complement measures are used, namely 
rectangular fit, roundness, elliptic fit, compactness, and shape index (corresponding explanations and formulae can be found in e.g., [31]-[33]). To characterize the (spatial) context the objects are embedded in, an individual gray-level value $\bar{c}_{O M P}(O)$ of an object $O$ with respect to an OMP is compared to the affiliated grey-level value $\bar{c}_{O M P}(U)$ of neighbor objects (Fig. 2). We compute the weighted mean difference $\bar{\Delta}_{O M P}$ between the grey-level values as follows:

$$
\bar{\Delta}_{O M P}(O)=\frac{1}{w} \sum_{U \in N_{O}} w_{U}\left(\bar{c}_{O M P}(O)-\bar{c}_{O M P}(U)\right)
$$

where $N_{O}$ is an adjacent neighbor object of $O$, and $w_{U}$ is the length of the common border between two objects, which serves as weight. Thereby, the range of feature values corresponds to

$$
\begin{gathered}
\text { MaxDev }=\max \left(\begin{array}{c}
\mid\left(\bar{c}_{O M P}(O)-\bar{c}_{O M P}\left(U_{1}\right) \mid, \ldots,\right. \\
\mid\left(\bar{c}_{O M P}(O)-\bar{c}_{O M P}\left(U_{i-1}\right)|,|\left(\bar{c}_{O M P}(O)-\bar{c}_{O M P}\left(U_{i}\right) \mid\right.\right.
\end{array}\right), \\
\bar{\Delta}_{O M P}(O) \in\left\{\mathbb{R} \mid-M a x D e v \leq \bar{\Delta}_{O M P}(O) \leq \text { MaxDev }\right\} .
\end{gathered}
$$

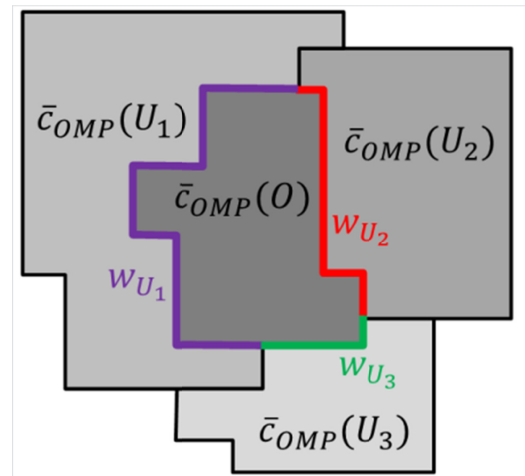

Fig. 2. Exemplified scheme for the computation of contextual features. The individual grey-level value $\bar{c}_{O M P}(O)$ of an object is compared to the grey-level values $\bar{c}_{O M P}(U)$ of neighbor objects. The length of a common border $w_{U}$ serves as weight for calculating the weighted mean difference of grey-level values.

It can be noted that this scheme allows also considering e.g., only the grey-level values of brighter or darker adjacent neighbor objects of $O$ and quantify the affiliated mean grey-level difference with respect to $O$. Overall, a quantification of contextual relations is primarily motivated by the circumstance that thematic classes can occur in (spatial) dependence of each other such as elevated objects and corresponding shadow areas. Finally, individual features can be stacked to a joint feature vector, which is subsequently fed to a supervised classification model.

\section{DATA SETS AND EXPERIMENTAL SETUP}

The experimental analysis was carried out by classifying two test areas taken from a VHR panchromatic image. This image was acquired over the city of Cologne, Germany, by the WorldView-II satellite sensor on $31^{\text {st }}$ January 2014 with a geometric resolution of $0.5 \mathrm{~m}$. The 
first data set is made up by $1000 \times 1000$ pixels and shows an urban area of Cologne, which is dominated by buildings of commercial use (Fig. 3(a); referred to as "data set 1 - commercial area"). The second data set comprises $902 \times 908$ pixels and represents an area of residential buildings next to the river Rhine (Fig. 3(d); referred to as "data set 2 - residential area"). Both image subsets feature a complex composition of urban land cover. Thereby, shadow areas can be observed primarily adjacent to buildings. In addition, the imagery represents an off-nadir acquisition. As such, facades of individual buildings can be identified in the direction of the sensor view. The pixels of the first image were grouped in five relevant thematic classes, namely "roof", "facade", "shadow", "vegetation", and "other impervious surface". The latter class comprises non-penetrable surfaces other than building-related ones such as roads or parking lots, which feature similar spectral characteristics. Data set 2 additionally features the thematic class "water". The thematic classes of pixels were determined based on photo-interpretation analysis under consideration of additional aerial imagery and cadastral maps. Varying configurations of corresponding labeled samples of data set 1 and 2 [Fig. 3(b-c) and (e-f)] were used for learning the models. In particular, five percent of all available labeled samples were drawn randomly in a stratified manner (i.e., in correspondence to the a priori probabilities of the classes) from the training data pool of the respective data set for ten different realizations. Thereby, generalization capabilities of the learned models are estimated based on a fivefold cross-validation procedure. 
data set 1 -commercial area

panchromatic image

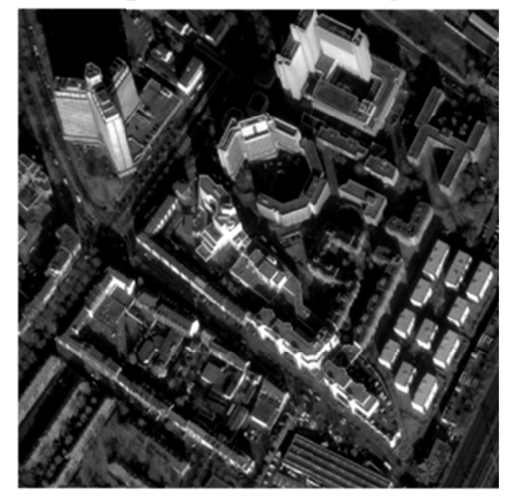

(a) labeled samples

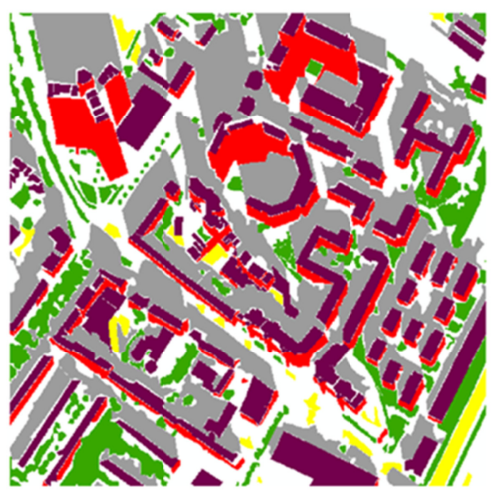

(b) number of labeled samples

\begin{tabular}{l|l}
\hline class & number \\
\hline vegetation & 26192 \\
\hline roof & 51069 \\
\hline facade & 25473 \\
\hline other impervious surface & 4967 \\
\hline shadow & 74244 \\
\hline$\Sigma$ & 181945 \\
\hline
\end{tabular}

(c)

\section{roof shadow vegetation}

facade other impervious surface

data set 2 -residential area

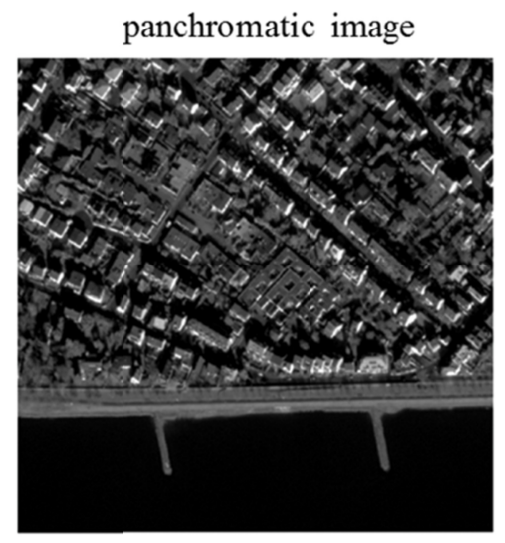

(d)

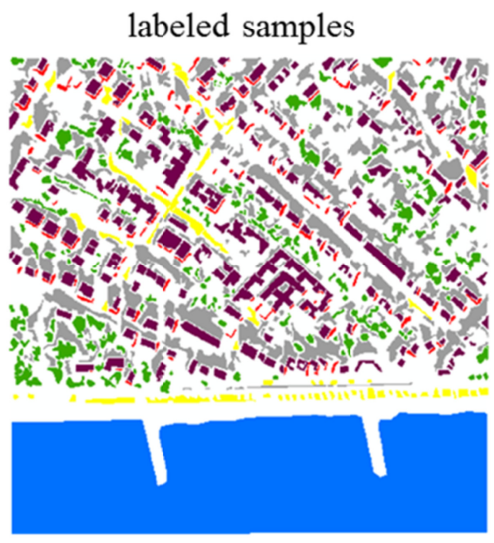

(e) number of labeled samples

\begin{tabular}{l|c}
\hline class & number \\
\hline vegetation & 8890 \\
\hline roof & 16226 \\
\hline facade & 2895 \\
\hline other impervious surface & 3710 \\
\hline shadow & 27360 \\
\hline water & 47240 \\
\hline$\Sigma$ & 106321 \\
\hline
\end{tabular}

(f)

\section{roof shadow $\square$ vegetation $\square$ water \\ facade other impervious surface}

Fig. 3. Experimental data. (a), (d) data sets from WorldView-II imagery acquired over the city of Cologne, Germany with available labeled samples (b-c; e-f).

For both images, we obtained the considered MOs (i.e., erosion, dilation, opening, closing, opening by top-hat, and closing by top-hat) based on a square-shaped SE with linear increasing size (i.e., 7, 11, 15, 19, 23). Since previous works showed that sets of mixed MOs can yield favorable results [3], the obtained sequences were stacked and considered as the benchmark vector in this work (denoted as $\left.M P(I)^{\text {stacked }}\right)$. Consequently, $M P(I)^{\text {stacked }}$ features 31 dimensions.

Regarding the computation of OMPs, we deploy a bottom-up region-growing segmentation algorithm for partition of $\boldsymbol{I}$ (i.e., fractal net evolution approach as implemented in the software environment eCognition [34], since it showed viable results in comparable settings [e.g., [35], [36]). In the experiments, we put more emphasis on shape heterogeneity rather than on gray-value heterogeneity with respect to the segmentation algorithm. This is due to the fact that manmade structures such as buildings and other elements of urban 
environments have distinct shape and size properties, unlike, for example, natural features. Analogously, the weights for heterogeneity of smoothness and compactness were maintained equal (i.e., shape: 0.7 , color: 0.5 ) and kept constant. Three hierarchical segmentation levels were considered for the experiments to account for objects with various sizes in the image. The scale parameters which determine - briefly speaking - the size of the objects, were chosen in a way that 1) at $s$, the majority of real-world objects are represented by several segments (i.e., over-segmentation); 2) at $s+1$, the majority of real-world objects are represented by a single affiliated segment; and 3 ) at $s+2$, the majority of real-world objects are represented in conjunction with other real-world objects by a single segment (i.e., under-segmentation) [37].

In accordance with the experimental setup of e.g., Dalla Murra et al. [14], we deploy a Random Forest (RF) approach [38] for evaluating the capabilities of the different profiles in modeling the characteristics of the respective imagery. This non-parametric classification approach was chosen to account for a considerable redundancy shown by the profiles (primarily induced by the consecutive window sizes and segmentation scales of the same morphological operations), which can be critical for the estimation of statistics in parametric approaches [14]. RF represents a decision-tree-based ensemble learning method for classification and regression. Such methods build a prediction model by utilizing the strength of a collection of simple base models. To this purpose, RF grows multiple decision trees on random subsets of the training data. The high variance among individual trees, letting each tree vote for the class assignment, and determining the respective class according to the majority of the votes, allows the accurate and robust classification of unlabeled samples, even when many noisy variables are existent [38], [39]. The hyperparameters that need to be determined for generating a RF model consist of the number of classification trees to be grown $n_{\text {tree }}$, and the number of features $m_{\text {try }}$ used at each node. To provide a reliable error estimate and maintaining the computation times in a reasonable range, we chose a $n_{\text {tree }}$ value of 500. This is in a good agreement with the RF parameter study performed by Genuer et al. [40]. According to Breiman [38], a value for $m_{t r y}=\sqrt{p}$, with $p$ denoting the number of input features, yields near optimum classification results. Thus, this heuristic was used to determine $m_{\text {try. }}$. To impose ceteris paribus-near conditions for comparison of the computed features a wrapper-based feature selection [41] was carried out to identify feature sets from the complete pool of available features, which allow for obtaining the best accuracies given the aforementioned experimental setup. Wrapper methods evaluate features by using accuracy estimates provided by the actual classification algorithm (here RF), which is deployed subsequent to feature selection. Thus, the classifier is trained and accuracy estimation is performed for each iteration of the evaluation process. Thereby, the respective models with the highest Kappa statistics ( $\kappa)$ - as primal measure for accuracy - were further considered. Such as strategy is very expensive from a computational point of view, however, it ensures most favorable model accuracy possible for a feature set, what is desirable in this comparative evaluation of features.

Thematic accuracies of the obtained maps (which are presented in Tables I and II) were assessed by computing global accuracy measures. We considered the weighted harmonic mean $\bar{F}$ of the F-measures (weighted by the cardinals of the thematic classes [42]), the overall accuracy (OA), and the $\kappa$ statistic based on the selected reference pixels (definition of those measures can be found in e.g., [43], [44]). Since overall accuracies of some models feature 
comparable levels, the statistical significance of the classification maps was additionally evaluated with the McNemar's test [43]. The test compares the classification outcomes for related samples (i.e., the number of miss-classified samples by the first model but not by the second model and the number of miss-classified samples by the second model but not by the first model) by assessing the standardized normal test statistic $Z$ for two thematic maps. The null hypothesis (i.e., the models feature the same error) can be rejected for an interval of significance $\alpha=5 \%$, if $|z|>1.96$. The results of this test are indicated in Tables I and II with the sign "*" when the accuracy of a model is higher and significantly different from the benchmark model (i.e., obtained with $M P(I)^{\text {stacked }}$ ).

\section{EXPERIMENTAL RESULTS AND DISCUSSION}

To understand the role of the different groups of features on the classification accuracy, systematic configurations of features were compiled and deployed for classification. Thereby, the different configurations of features were fed to the wrapper-based feature selection scheme described above to eventually prune irrelevant features and ensure most favorable model accuracy possible.

\section{A. Data set 1 - commercial area}

The results of this analysis for the data set of the commercial area are documented in Table I in terms of accuracy measures. Selected results are also visualized in Fig. 4. Generally, the results in Table I are differentiated according to the pixel and object-based approaches and with respect to the latter in dependence of the deployed aggregation function.

First of all, it can be noticed that the model based on the individual pixels of the panchromatic band does not feature viable accuracies and the affiliated classification map is not spatially consistent at all [Fig. 4(a)]. Instead, the usage of $M P(I)^{\text {stacked }}$ allows for obtaining a considerably improved level of accuracy and a classification map with definite spatial consistency [Fig. 4(b)]. Regarding the deployment of OBIA techniques, notably, a significant improvement in terms of accuracies can be achieved in this example already with respect to $M P(I)^{\text {stacked }}$, when using the object-based representation of the non-transformed image for model learning (i.e., an average increase of more than $7 \%$ in terms of $\kappa$ statistic can be observed independent of the chosen aggregation function). As can be seen from [Fig. 4(c)], spatial assemblages of classes are less fragmented compared to the previous result, however, overgeneralization also occurs. The learning of models based on $\operatorname{OMP}(\boldsymbol{I})^{\text {stacked }}$ provides solutions with a further substantial increase of accuracy (i.e., an average increase of more than $4 \%$ and $12 \%$ in terms of $\kappa$ statistic can be observed with respect to the object-based representation of the non-transformed image and $M P(I)^{\text {stacked }}$, respectively, independent of the chosen aggregation function). Thereby, the affiliated classification map [Fig. 4(d)] internalizes two favorable aspects, as the solution is clearly less fragmented than $M P(I)^{\text {stacked }}$ and the level of overgeneralization remains low. 
TABLE I

Study Area 1 (COMmercial AREa): Classification ACCURACies ObTained with Different Feature Vectors REPORTED AS MEAN AND STANDARD DEVIATION (IN BRACKETS) FROM TEN REALIZATIONS WITH A VARYING CONFIGURATION OF LABELED SAMPles. “*” = SignifiCANT DiFFERENCE FOR AN INTERVAL OF SigNifiCANCE OF A $=5 \%$ FROM THE BENCHMARK FEATURE VECTOR (I.E., $M P(I)^{\text {stacked }}$ ) AS EVALUATED WITH MCNEMAR'S TEST AND

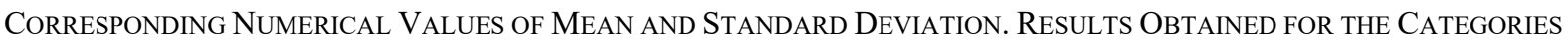
“PIXEL-BASED” AND “OBJECT-BASED - MEAN” ARE ALSO ViSUALIZED IN FigURE 4

\begin{tabular}{|c|c|c|c|c|c|}
\hline \multirow{2}{*}{\multicolumn{2}{|c|}{ feature vector }} & \multicolumn{3}{|c|}{ accuracy measures } & \multirow{4}{*}{$\begin{array}{c}\text { McNemar's } \\
\text { test }\end{array}$} \\
\hline & & \multirow{2}{*}{$\begin{array}{c}\overline{\mathrm{F}}(\%) \\
42.31 \\
( \pm 4.55)\end{array}$} & \multirow{2}{*}{$\begin{array}{c}\text { OA }(\%) \\
66.86 \\
( \pm 0.21)\end{array}$} & \multirow{2}{*}{$\begin{array}{c}\kappa(\%) \\
52.92 \\
( \pm 0.07)\end{array}$} & \\
\hline pixel- & $I$ & & & & \\
\hline based & $M P(I)^{\text {stacked }}$ & $\begin{array}{r}84.96 \\
( \pm 0.29) \\
\end{array}$ & $\begin{array}{r}86.09 \\
( \pm 0.21) \\
\end{array}$ & $\begin{array}{c}80.36 \\
( \pm 0.36) \\
\end{array}$ & \\
\hline \multirow{6}{*}{$\begin{array}{l}\text { object- } \\
\text { based - } \\
\text { mean }\end{array}$} & $\boldsymbol{I}_{\Psi, \bar{x}}$ & $\begin{array}{c}91.80 \\
( \pm 0.18) \\
\end{array}$ & $\begin{array}{c}91.86 \\
( \pm 0.18) \\
\end{array}$ & $\begin{array}{c}88.62 \\
( \pm 0.29) \\
\end{array}$ & $\begin{array}{c}*, 19.84 \\
( \pm 0.04)\end{array}$ \\
\hline & $O M P(I)_{\bar{x}}^{\text {stacked }}$ & $\begin{array}{c}94.78 \\
( \pm 0.08) \\
\end{array}$ & $\begin{array}{c}94.80 \\
( \pm 0.07) \\
\end{array}$ & $\begin{array}{r}92.72 \\
( \pm 0.12) \\
\end{array}$ & $\begin{array}{c}*, 27.03 \\
( \pm 0.51)\end{array}$ \\
\hline & $O M P(I)_{\bar{x}}^{\text {stacked }}, \boldsymbol{I}_{\Psi}^{\text {shape }}$ & $\begin{array}{c}94.87 \\
( \pm 0.07)\end{array}$ & $\begin{array}{c}94.88 \\
( \pm 0.08)\end{array}$ & $\begin{array}{c}92.84 \\
( \pm 0.09)\end{array}$ & $\begin{array}{c}*, 27.19 \\
( \pm 0.63)\end{array}$ \\
\hline & $O M P(\boldsymbol{I})_{\bar{x}}^{\text {stacked }}, O M P(\boldsymbol{I})_{\bar{x}}^{\text {context }}$ & $\begin{array}{c}94.78 \\
( \pm 0.08) \\
\end{array}$ & $\begin{array}{c}94.83 \\
( \pm 0.06)\end{array}$ & $\begin{array}{c}92.74 \\
( \pm 0.11) \\
\end{array}$ & $\begin{array}{c}*, 27.08 \\
( \pm 0.56)\end{array}$ \\
\hline & $O M P(\boldsymbol{I})_{\bar{x}}^{\text {stacked }}, \boldsymbol{I}_{\Psi}^{\text {shape }}, O M P(\boldsymbol{I})_{\bar{x}}^{\text {context }}$ & $\begin{array}{c}94.93 \\
( \pm 0.03)\end{array}$ & $\begin{array}{c}94.94 \\
( \pm 0.04)\end{array}$ & $\begin{array}{c}92.94 \\
( \pm 0.04)\end{array}$ & $\begin{array}{c}*, 27.20 \\
( \pm 0.62)\end{array}$ \\
\hline & $O M P(\boldsymbol{I})_{\bar{x}}^{\text {stacked }}, \boldsymbol{I}_{\Psi}^{\text {shape }}, O M P(\boldsymbol{I})_{\bar{x}}^{\text {context }}, M P(\boldsymbol{I})^{\text {stacked }}$ & $\begin{array}{c}94.97 \\
( \pm 0.02) \\
\end{array}$ & $\begin{array}{r}95.02 \\
( \pm 0.03) \\
\end{array}$ & $\begin{array}{c}93.01 \\
( \pm 0.02) \\
\end{array}$ & $\begin{array}{c}*, 27.29 \\
( \pm 0.69) \\
\end{array}$ \\
\hline \multirow{6}{*}{$\begin{array}{l}\text { object- } \\
\text { based - } \\
\text { mode }\end{array}$} & $\boldsymbol{I}_{\Psi, M O}$ & $\begin{array}{c}90.77 \\
( \pm 0.04)\end{array}$ & $\begin{array}{c}90.91 \\
( \pm 0.06)\end{array}$ & $\begin{array}{c}87.41 \\
( \pm 0.12) \\
\end{array}$ & $\begin{array}{c}*, 17.44 \\
( \pm 0.69)\end{array}$ \\
\hline & $O M P(I)_{M O}^{\text {stacked }}$ & $\begin{array}{c}94.87 \\
( \pm 0.17) \\
\end{array}$ & $\begin{array}{c}94.89 \\
( \pm 0.15)\end{array}$ & $\begin{array}{c}92.85 \\
( \pm 0.24) \\
\end{array}$ & $\begin{array}{c}*, 27.39 \\
( \pm 0.24)\end{array}$ \\
\hline & $O M P(\boldsymbol{I})_{M O}^{\text {stacked }}, \boldsymbol{I}_{\Psi}^{\text {shape }}$ & $\begin{array}{c}94.99 \\
( \pm 0.05) \\
\end{array}$ & $\begin{array}{c}95.01 \\
( \pm 0.04)\end{array}$ & $\begin{array}{c}93.01 \\
( \pm 0.08) \\
\end{array}$ & $\begin{array}{c}*, 27.55 \\
( \pm 0.39)\end{array}$ \\
\hline & $O M P(\boldsymbol{I})_{M O}^{\text {stacked }}, O M P(\boldsymbol{I})_{M O}^{\text {context }}$ & $\begin{array}{c}94.87 \\
( \pm 0.17) \\
\end{array}$ & $\begin{array}{r}94.89 \\
( \pm 0.15) \\
\end{array}$ & $\begin{array}{c}92.85 \\
( \pm 0.24) \\
\end{array}$ & $\begin{array}{c}*, 27.44 \\
( \pm 0.28)\end{array}$ \\
\hline & $O M P(\boldsymbol{I})_{M O}^{\text {stacked }}, \boldsymbol{I}_{\Psi}^{\text {shape }}, O M P(\boldsymbol{I})_{M O}^{\text {context }}$ & $\begin{array}{c}95.04 \\
( \pm 0.12) \\
\end{array}$ & $\begin{array}{c}95.04 \\
( \pm 0.09) \\
\end{array}$ & $\begin{array}{c}93.02 \\
( \pm 0.07) \\
\end{array}$ & $\begin{array}{c}*, 27.58 \\
( \pm 0.41)\end{array}$ \\
\hline & $O M P(\boldsymbol{I})_{M O}^{\text {stacked }}, \boldsymbol{I}_{\Psi}^{\text {shape }}, O M P(\boldsymbol{I})_{M O}^{\text {context }}, M P(\boldsymbol{I})^{\text {stacked }}$ & $\begin{array}{r}95.13 \\
( \pm 0.13) \\
\end{array}$ & $\begin{array}{r}95.05 \\
( \pm 0.06) \\
\end{array}$ & $\begin{array}{c}93.08 \\
( \pm 0.05) \\
\end{array}$ & $\begin{array}{c}*, 27.62 \\
( \pm 0.47) \\
\end{array}$ \\
\hline \multirow{6}{*}{$\begin{array}{l}\text { object- } \\
\text { based - } \\
\text { median }\end{array}$} & $\boldsymbol{I}_{\psi, \tilde{x}}$ & $\begin{array}{c}91.17 \\
( \pm 0.05)\end{array}$ & $\begin{array}{c}91.29 \\
( \pm 0.05)\end{array}$ & $\begin{array}{c}87.79 \\
( \pm 0.03) \\
\end{array}$ & $\begin{array}{c}*, 19.30 \\
( \pm 0.16)\end{array}$ \\
\hline & $O M P(\boldsymbol{I})_{\tilde{x}}^{\text {stacked }}$ & $\begin{array}{c}94.71 \\
( \pm 0.03)\end{array}$ & $\begin{array}{c}94.74 \\
( \pm 0.02)\end{array}$ & $\begin{array}{c}92.63 \\
( \pm 0.04)\end{array}$ & $\begin{array}{c}*, 26.86 \\
( \pm 0.21)\end{array}$ \\
\hline & $O M P(\boldsymbol{I})_{\tilde{x}}^{\text {stacked }}, \boldsymbol{I}_{\Psi}^{\text {shape }}$ & $\begin{array}{c}94.84 \\
( \pm 0.03) \\
\end{array}$ & $\begin{array}{r}94.89 \\
( \pm 0.05) \\
\end{array}$ & $\begin{array}{r}92.76 \\
( \pm 0.02) \\
\end{array}$ & $\begin{array}{l}*, 27.65 \\
( \pm 0.09)\end{array}$ \\
\hline & $O M P(\boldsymbol{I})_{\tilde{x}}^{\text {stacked }}, O M P(\boldsymbol{I})_{\tilde{x}}^{\text {context }}$ & $\begin{array}{c}94.72 \\
( \pm 0.06) \\
\end{array}$ & $\begin{array}{c}94.77 \\
( \pm 0.04) \\
\end{array}$ & $\begin{array}{c}92.63 \\
( \pm 0.04) \\
\end{array}$ & $\begin{array}{c}*, 27.40 \\
( \pm 0.08)\end{array}$ \\
\hline & $O M P(\boldsymbol{I})_{\tilde{x}}^{\text {stacked }}, \boldsymbol{I}_{\Psi}^{\text {shape }}, O M P(\boldsymbol{I})_{\tilde{x}}^{\text {context }}$ & $\begin{array}{c}94.88 \\
( \pm 0.09)\end{array}$ & $\begin{array}{r}95.02 \\
( \pm 0.02)\end{array}$ & $\begin{array}{c}92.90 \\
( \pm 0.09)\end{array}$ & $\begin{array}{l}*, 27.74 \\
( \pm 0.09)\end{array}$ \\
\hline & $O M P(\boldsymbol{I})_{\tilde{x}}^{\text {stacked }}, \boldsymbol{I}_{\Psi}^{\text {shape }}, O M P(\boldsymbol{I})_{\tilde{x}}^{\text {context }}, M P(\boldsymbol{I})^{\text {stacked }}$ & $\begin{array}{r}94.89 \\
( \pm 0.04) \\
\end{array}$ & $\begin{array}{r}95.04 \\
( \pm 0.03) \\
\end{array}$ & $\begin{array}{r}93.05 \\
( \pm 0.05) \\
\end{array}$ & $\begin{array}{c}*, 27.82 \\
( \pm 0.04) \\
\end{array}$ \\
\hline
\end{tabular}

The encoding of shape and context-related properties enables an additional slight increase of model accuracies compared to $O M P(I)^{\text {stacked }}[$ Fig. 4(e)-(g)]. Thereby, the shape-related features are more valuable in this example than the context-related ones, since the increase of accuracy is consistently a little higher. A joint consideration of both groups of features allows for obtaining further increased accuracies. This indicates that both groups of features can 
encode additional discriminative information compared to the solely application of both MPs or OMPs. Lastly, the creation of feature vectors containing all features allowed for further slight improvements and ensures the highest accuracies observed during all runs.

A clear recommendation regarding the most favorable aggregation function cannot be drawn from this example. This is evident since the most favorable feature vectors of different categories are attributed to different aggregation functions. However, the results do not vary greatly between the different aggregation functions and the overall accuracy pattern is consistent - independent of the chosen aggregation function. Overall, feature vectors based on OMPs reveal more favorable results than models obtained with MPs, what demonstrates the viability of this concept. 
labeled reference pixels
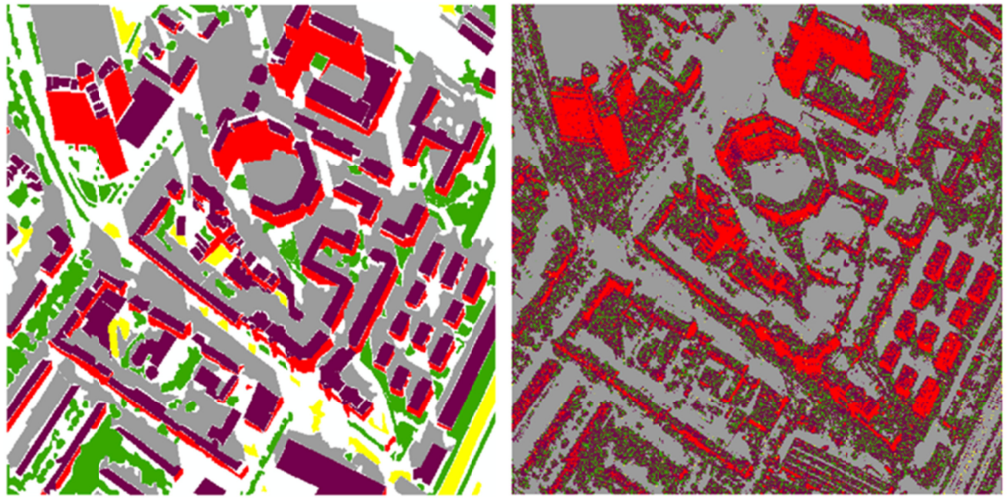

(a)

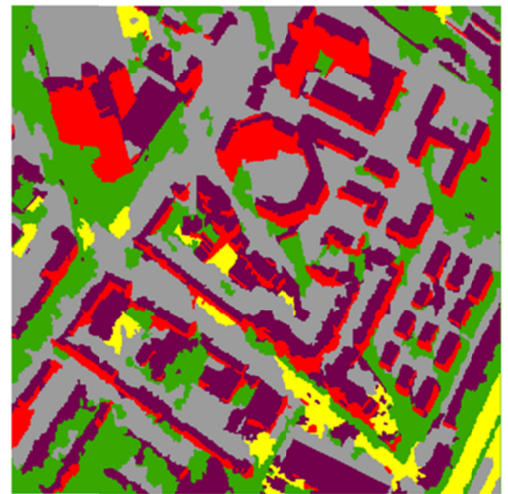

(c)

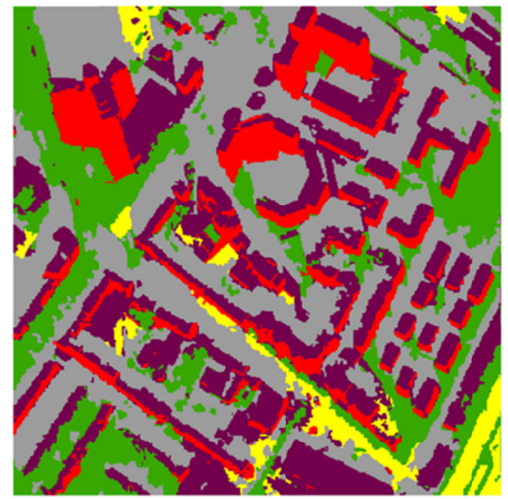

(f)

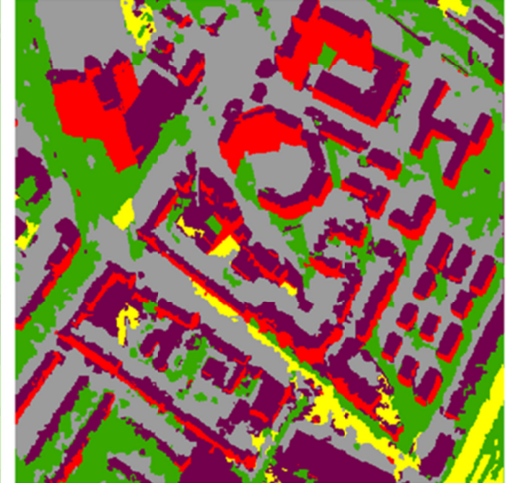

(d)

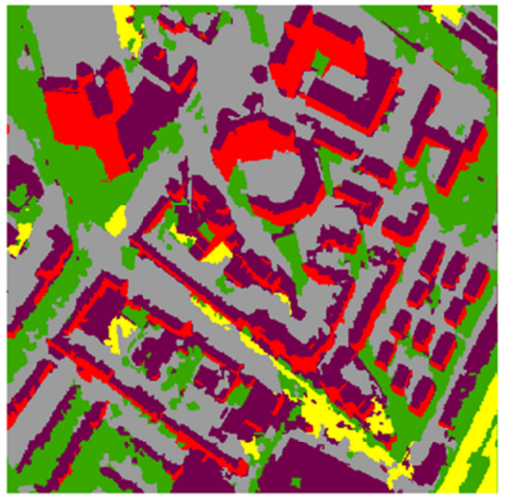

(g)

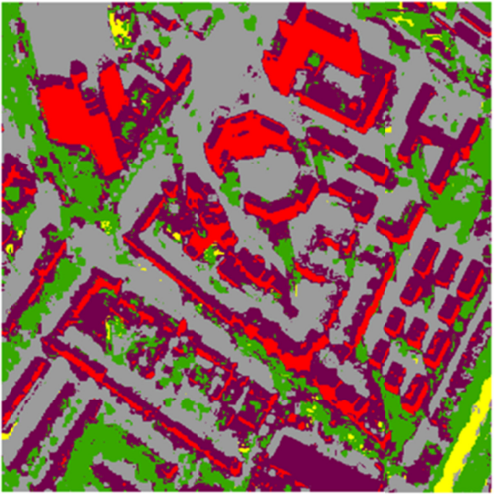

(b)

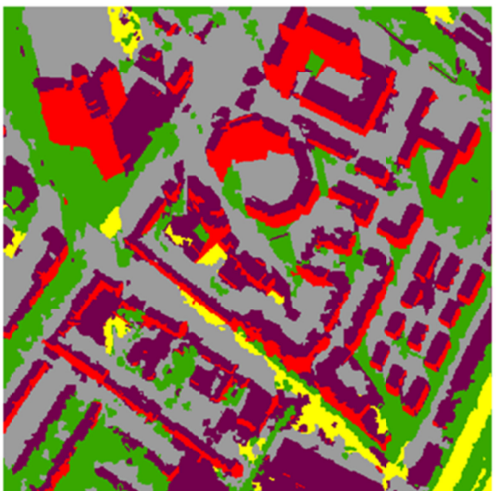

(e)

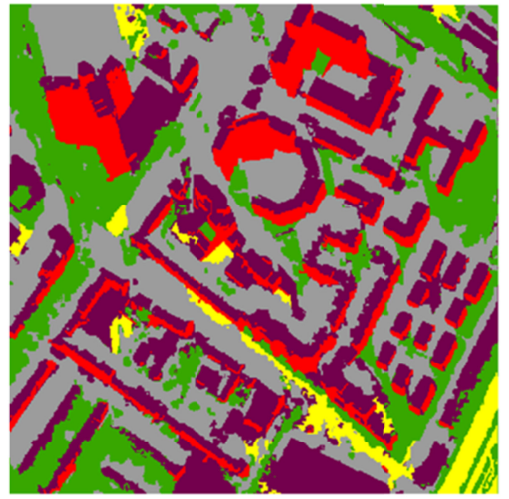

(h)

roof facade other impervious surface $\quad$ shadow vegetation

Fig. 4. Classification results obtained with different feature vectors for the commercial area. (a) pixel-based nontransformed panchromatic imagery; (b) $M P(\boldsymbol{I})^{\text {stacked }}$; $\boldsymbol{I}_{\Psi, \bar{x}}$, (object-based non-transformed panchromatic imagery based on mean); (d) $O M P(\boldsymbol{I})_{\bar{x}}^{\text {stacked }}$; (e) $O M P(\boldsymbol{I})_{\bar{x}}^{\text {stacked }}$ under additional consideration of shape features; (f) $O M P(I)_{\bar{x}}^{\text {stacked }}$ under additional consideration of context features; (g) $O M P(I)_{\bar{x}}^{\text {stacked }}$ under additional consideration of both shape and context features; (h) same as (g) but additional consideration of $M P(I)^{\text {stacked }}$. 


\section{B. Data set 2 - residential area}

The classification accuracies for the data set of the residential area are documented in Table II and the application of selected models can be found in Fig. 5. In accordance with the results from data set 1 , the information of the single panchromatic image cannot reliably partition the pixels in thematic classes [Fig. 5(a)]. Again, the use of MPs enables a steep increase of accuracy measures (e.g., from $61 \%$ to $93 \%$ in terms of $\kappa$ statistic) and a stable classification map with respect to its spatial consistency [Fig. 5(b)]. An improvement can be obtained also for this example when relying on the object-based representation of the non-transformed image for model learning [Fig 5(c)] compared to the outcomes achieved with the single panchromatic image. However, in contrast to the previous example, this model features lower accuracies compared to $M P(I)^{\text {stacked }}$. Instead, the postulated OMPs exceed the accuracy of the benchmark vector considerably again (i.e., an average increase of more than $4 \%$ in terms of $\kappa$ statistic can be observed independent of the chosen aggregation function), although the levels of model accuracies are already very high. Moreover, as for the previous data set, the considered shape and context-related features allow for a further increase of model performance. In accordance, the highest accuracies can be retrieved based on all available features. 
TABLE II

Study Area 2 (Commercial Area): Classification ACCuracies Obtained with Different Feature Vectors REPORTED AS MEAN AND STANDARD DEVIATION (IN BRACKETS) FROM TEN REALIZATIONS WiTH A VARYING CONFIGURATION OF LABELED SAMPLES. “*”= SigNifiCANT DifFERENCE FOR AN INTERVAL OF SigNifiCANCE OF A $=5 \%$ FROM THE BENCHMARK FEATURE VECTOR (I.E., $M P(\boldsymbol{I})^{\text {stacked }}$ ) AS EVALUATED WITH MCNEMAR'S TEST AND

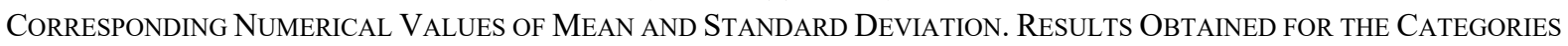
“PIXEL-BASED” AND “OBJECT-BASED - MEAN” ARE ALSO VISUALIZED IN FIGURE 4

\begin{tabular}{|c|c|c|c|c|c|}
\hline \multirow{2}{*}{\multicolumn{2}{|c|}{ feature vector }} & \multicolumn{3}{|c|}{ accuracy measures } & \multirow{4}{*}{$\begin{array}{c}\text { McNemar's } \\
\text { test }\end{array}$} \\
\hline & & \multirow{2}{*}{$\begin{array}{c}\overline{\mathbf{F}}(\%) \\
61.25 \\
( \pm 0.18)\end{array}$} & \multirow{2}{*}{$\begin{array}{c}\mathrm{OA}(\%) \\
72.92 \\
( \pm 0.06)\end{array}$} & \multirow{2}{*}{$\begin{array}{c}\kappa(\%) \\
60.90 \\
( \pm 0.23) \\
\end{array}$} & \\
\hline pixel- & $I$ & & & & \\
\hline based & $M P(I)^{\text {stacked }}$ & $\begin{array}{r}93.95 \\
( \pm 0.02) \\
\end{array}$ & $\begin{array}{r}94.90 \\
( \pm 0.04) \\
\end{array}$ & $\begin{array}{r}92.77 \\
( \pm 0.01) \\
\end{array}$ & \\
\hline \multirow{6}{*}{$\begin{array}{l}\text { object- } \\
\text { based - } \\
\text { mean }\end{array}$} & $\boldsymbol{I}_{\Psi, \bar{x}}$ & $\begin{array}{c}94.69 \\
( \pm 0.04) \\
\end{array}$ & $\begin{array}{c}94.64 \\
( \pm 0.03) \\
\end{array}$ & $\begin{array}{c}92.37 \\
( \pm 0.05) \\
\end{array}$ & - \\
\hline & $O M P(I)_{\bar{x}}^{\text {stacked }}$ & $\begin{array}{c}97.71 \\
( \pm 0.12) \\
\end{array}$ & $\begin{array}{c}97.77 \\
( \pm 0.12) \\
\end{array}$ & $\begin{array}{c}96.85 \\
( \pm 0.16) \\
\end{array}$ & $\begin{array}{l}*, 10.79 \\
( \pm 0.20)\end{array}$ \\
\hline & $O M P(\boldsymbol{I})_{\bar{x}}^{\text {stacked }}, \boldsymbol{I}_{\Psi}^{\text {shape }}$ & $\begin{array}{c}97.82 \\
( \pm 0.03) \\
\end{array}$ & $\begin{array}{c}97.89 \\
( \pm 0.05) \\
\end{array}$ & $\begin{array}{c}97.01 \\
( \pm 0.05) \\
\end{array}$ & $\begin{array}{l}*, 11.17 \\
( \pm 0.10)\end{array}$ \\
\hline & $O M P(I)_{\bar{x}}^{\text {stacked }}, O M P(I)_{\bar{x}}^{\text {context }}$ & $\begin{array}{c}97.86 \\
( \pm 0.04) \\
\end{array}$ & $\begin{array}{c}97.92 \\
( \pm 0.05) \\
\end{array}$ & $\begin{array}{c}97.06 \\
( \pm 0.05) \\
\end{array}$ & $\begin{array}{l}*, 11.17 \\
( \pm 0.04)\end{array}$ \\
\hline & $O M P(\boldsymbol{I})_{\bar{x}}^{\text {stacked }}, \boldsymbol{I}_{\Psi}^{\text {shape }}, O M P(\boldsymbol{I})_{\bar{x}}^{\text {context }}$ & $\begin{array}{c}98.00 \\
( \pm 0.10)\end{array}$ & $\begin{array}{c}98.05 \\
( \pm 0.08) \\
\end{array}$ & $\begin{array}{c}97.25 \\
( \pm 0.14) \\
\end{array}$ & $\begin{array}{l}*, 11.40 \\
( \pm 0.27)\end{array}$ \\
\hline & $O M P(\boldsymbol{I})_{\bar{x}}^{\text {stacked }}, \boldsymbol{I}_{\Psi}^{\text {shape }}, O M P(\boldsymbol{I})_{\bar{x}}^{\text {context }}, M P(\boldsymbol{I})^{\text {stacked }}$ & $\begin{array}{c}98.36 \\
( \pm 0.01) \\
\end{array}$ & $\begin{array}{r}98.39 \\
( \pm 0.01) \\
\end{array}$ & $\begin{array}{r}97.43 \\
( \pm 0.32) \\
\end{array}$ & $\begin{array}{c}*, 13.28 \\
( \pm 0.08) \\
\end{array}$ \\
\hline \multirow{6}{*}{$\begin{array}{l}\text { object- } \\
\text { based - } \\
\text { mode }\end{array}$} & $\boldsymbol{I}_{\Psi, M O}$ & $\begin{array}{c}93.29 \\
( \pm 0.44)\end{array}$ & $\begin{array}{c}93.91 \\
( \pm 0.33)\end{array}$ & $\begin{array}{c}91.37 \\
( \pm 0.52)\end{array}$ & - \\
\hline & $O M P(I)_{M O}^{\text {stacked }}$ & $\begin{array}{c}97.89 \\
( \pm 0.04) \\
\end{array}$ & $\begin{array}{c}97.95 \\
( \pm 0.04) \\
\end{array}$ & $\begin{array}{c}97.10 \\
( \pm 0.07)\end{array}$ & $\begin{array}{l}*, 10.71 \\
( \pm 0.93)\end{array}$ \\
\hline & $O M P(\boldsymbol{I})_{M O}^{\text {stacked }}, \boldsymbol{I}_{\Psi}^{\text {shape }}$ & $\begin{array}{c}97.94 \\
( \pm 0.05) \\
\end{array}$ & $\begin{array}{c}97.99 \\
( \pm 0.03)\end{array}$ & $\begin{array}{c}97.16 \\
( \pm 0.01) \\
\end{array}$ & $\begin{array}{c}*, 10.89 \\
( \pm 0.75)\end{array}$ \\
\hline & $O M P(\boldsymbol{I})_{M O}^{\text {stacked }}, O M P(\boldsymbol{I})_{M O}^{\text {context }}$ & $\begin{array}{c}97.92 \\
( \pm 0.02)\end{array}$ & $\begin{array}{c}97.98 \\
( \pm 0.01)\end{array}$ & $\begin{array}{c}97.14 \\
( \pm 0.03)\end{array}$ & $\begin{array}{c}*, 10.89 \\
( \pm 0.75)\end{array}$ \\
\hline & $O M P(\boldsymbol{I})_{M O}^{\text {stacked }}, \boldsymbol{I}_{\Psi}^{\text {shape }}, O M P(\boldsymbol{I})_{M O}^{\text {context }}$ & $\begin{array}{r}97.94 \\
( \pm 0.01) \\
\end{array}$ & $\begin{array}{r}97.99 \\
( \pm 0.02) \\
\end{array}$ & $\begin{array}{c}97.16 \\
( \pm 0.01) \\
\end{array}$ & $\begin{array}{c}*, 10.91 \\
( \pm 0.73) \\
\end{array}$ \\
\hline & $O M P(\boldsymbol{I})_{M O}^{\text {stacked }}, \boldsymbol{I}_{\Psi}^{\text {shape }}, O M P(\boldsymbol{I})_{M O}^{\text {context }}, M P(\boldsymbol{I})^{\text {stacked }}$ & $\begin{array}{r}98.37 \\
( \pm 0.09) \\
\end{array}$ & $\begin{array}{r}98.40 \\
( \pm 0.09) \\
\end{array}$ & $\begin{array}{r}97.74 \\
( \pm 0.14) \\
\end{array}$ & $\begin{array}{c}*, 12.60 \\
( \pm 0.88) \\
\end{array}$ \\
\hline \multirow{6}{*}{$\begin{array}{l}\text { object- } \\
\text { based - } \\
\text { median }\end{array}$} & $\boldsymbol{I}_{\Psi, \tilde{x}}$ & $\begin{array}{c}93.43 \\
( \pm 0.10) \\
\end{array}$ & $\begin{array}{c}93.98 \\
( \pm 0.05) \\
\end{array}$ & $\begin{array}{c}91.46 \\
( \pm 0.05)\end{array}$ & - \\
\hline & $O M P(I)_{\tilde{x}}^{\text {stacked }}$ & $\begin{array}{c}97.74 \\
( \pm 0.13)\end{array}$ & $\begin{array}{c}97.82 \\
( \pm 0.13)\end{array}$ & $\begin{array}{c}96.92 \\
( \pm 0.16)\end{array}$ & $\begin{array}{l}*, 10.49 \\
( \pm 0.03)\end{array}$ \\
\hline & $O M P(\boldsymbol{I})_{\tilde{x}}^{\text {stacked }}, \boldsymbol{I}_{\Psi}^{\text {shape }}$ & $\begin{array}{c}97.88 \\
( \pm 0.06) \\
\end{array}$ & $\begin{array}{c}97.94 \\
( \pm 0.07) \\
\end{array}$ & $\begin{array}{c}97.09 \\
( \pm 0.08) \\
\end{array}$ & $\begin{array}{c}*, 10.96 \\
( \pm 0.26)\end{array}$ \\
\hline & $O M P(\boldsymbol{I})_{\tilde{x}}^{\text {stacked }}, O M P(\boldsymbol{I})_{\tilde{x}}^{\text {context }}$ & $\begin{array}{c}98.01 \\
( \pm 0.14)\end{array}$ & $\begin{array}{c}98.07 \\
( \pm 0.12)\end{array}$ & $\begin{array}{c}97.27 \\
( \pm 0.19)\end{array}$ & $\begin{array}{c}*, 11.26 \\
( \pm 0.81)\end{array}$ \\
\hline & $O M P(\boldsymbol{I})_{\tilde{x}}^{\text {stacked }}, \boldsymbol{I}_{\Psi}^{\text {shape }}, O M P(\boldsymbol{I})_{\tilde{x}}^{\text {context }}$ & $\begin{array}{c}98.06 \\
( \pm 0.08) \\
\end{array}$ & $\begin{array}{c}98.11 \\
( \pm 0.08) \\
\end{array}$ & $\begin{array}{r}97.32 \\
( \pm 0.13) \\
\end{array}$ & $\begin{array}{l}*, 11.30 \\
( \pm 0.77)\end{array}$ \\
\hline & $O M P(\boldsymbol{I})_{\tilde{x}}^{\text {stacked }}, \boldsymbol{I}_{\psi}^{\text {shape }}, O M P(\boldsymbol{I})_{\tilde{x}}^{\text {context }}, M P(\boldsymbol{I})^{\text {stacked }}$ & $\begin{array}{r}98.26 \\
( \pm 0.08) \\
\end{array}$ & $\begin{array}{r}98.32 \\
( \pm 0.08) \\
\end{array}$ & $\begin{array}{r}97.62 \\
( \pm 0.13) \\
\end{array}$ & $\begin{array}{l}*, 12.29 \\
( \pm 0.86)\end{array}$ \\
\hline
\end{tabular}

As can be seen from the visualization of the OMP-based models [Fig 5(d)-(h)], which all exceed the accuracy of the baseline vector, the obtained maps are not only spatially less fragmented again but also some thematic classes are in general extracted way more reliably. This can be prominently observed for the thematic class "other impervious surface", which 
can be retrieved in this example with a significantly decreased error of omission (i.e., the mean recall rate of the models based on $M P(I)^{\text {stacked }}$ is 0.552 for this class, whereas the mean recall rate of the models based on the OMP-processing corresponds to 0.882). Again, a universal superiority with respect to one particular aggregation function cannot be observed, however, the overall accuracy patterns is consistent. Finally, it can be noted that OMPs and affiliated feature vectors enabled learning of models which exhibit a significant increased accuracy compared to standard MPs again.
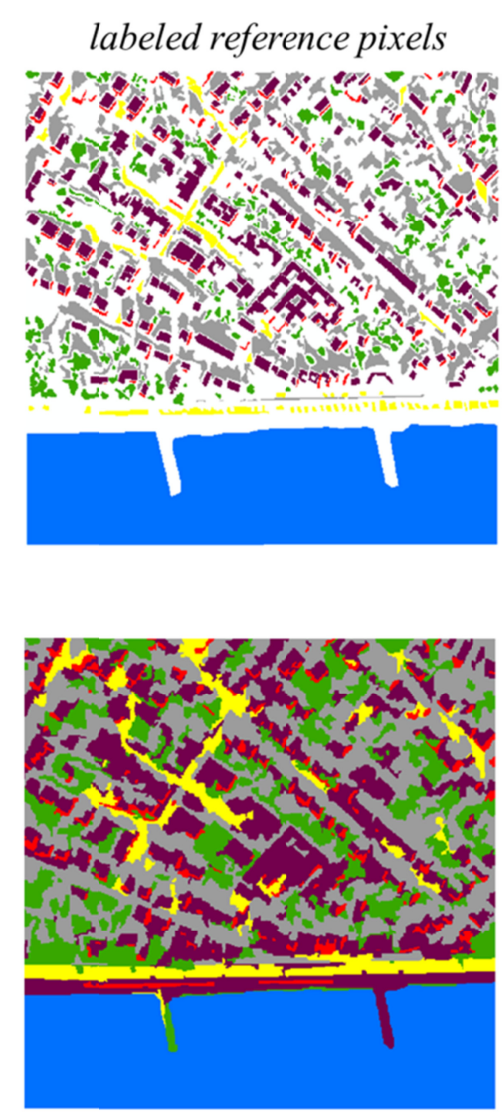

(c)

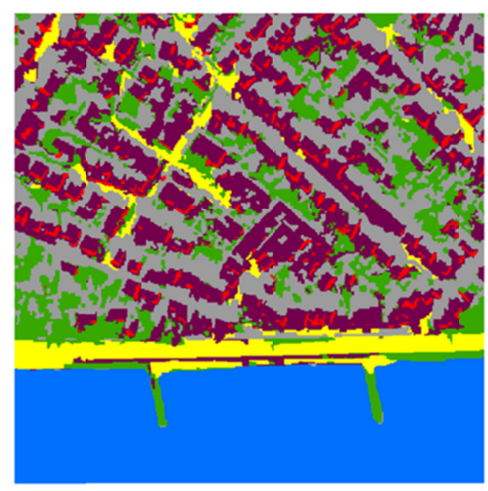

(f)

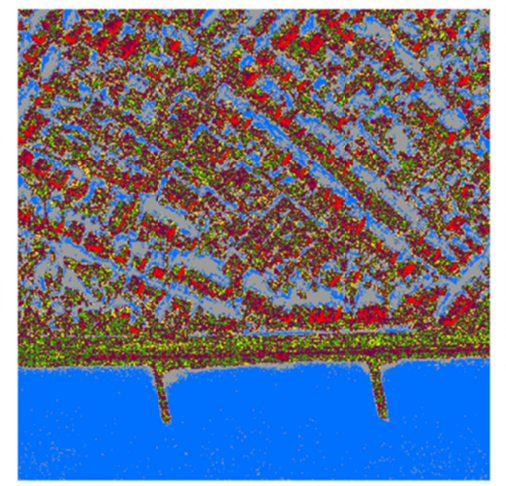

(a)

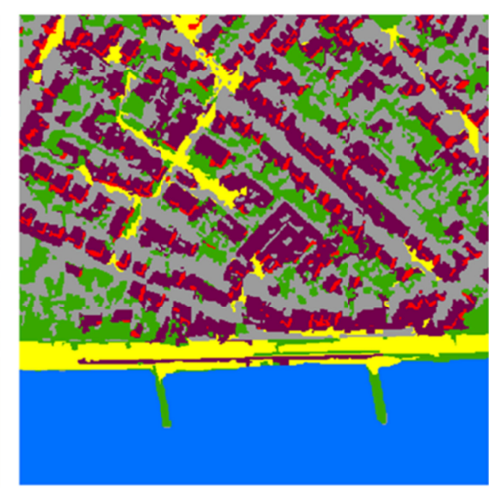

(d)

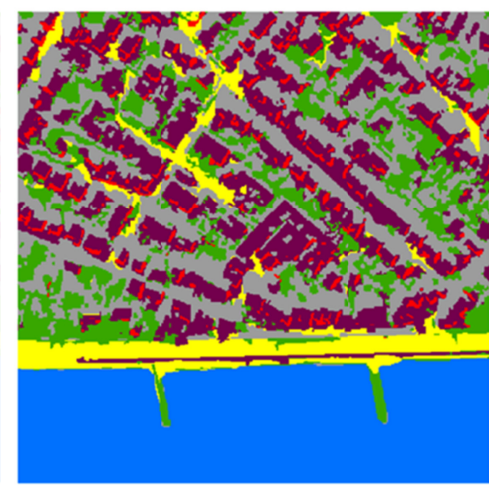

(g)

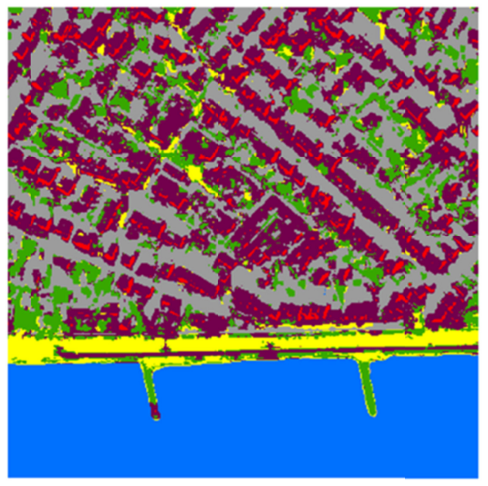

(b)

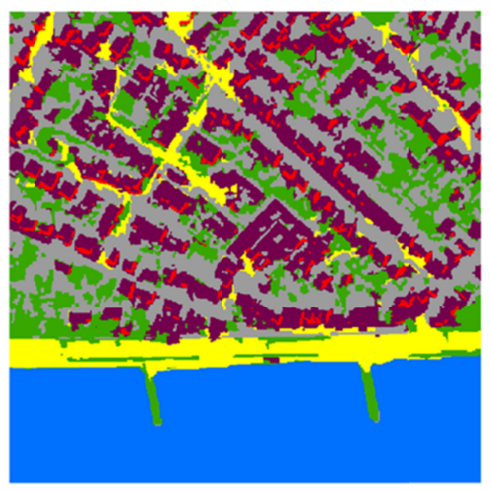

(e)

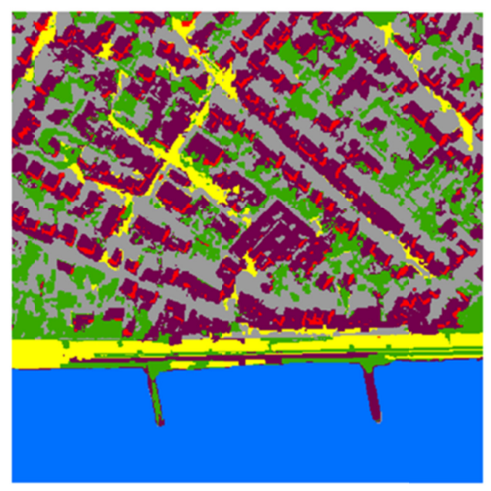

(h)

roof $\square$ facade $\square$ other impervious surface $\square$ shadow $\square$ vegetation $\square$ water

Fig. 5. Classification results obtained with different feature vectors for the residential area. (a) pixel-based nontransformed panchromatic imagery; $M P(I)^{\text {stacked }}$ $\boldsymbol{I}_{\Psi, \bar{x}}, \quad$ (object-based non-transformed panchromatic imagery based on mean); (d) 
$O M P(\boldsymbol{I})_{\bar{x}}^{\text {stacked }}$; (e) $O M P(\boldsymbol{I})_{\bar{x}}^{\text {stacked }}$ under additional consideration of shape features; (f) $O M P(I)_{\bar{x}}^{\text {stacked }}$ under additional consideration of context features; (g) $O M P(I)_{\bar{x}}^{\text {stacked }}$ under additional consideration of both shape and context features; $(\mathrm{h})$ same as $(\mathrm{g})$ but additional consideration of $M P(I)^{\text {stacked }}$.

\section{CONCLUSIONS AND OUTLOOK}

In this paper, the concept of object-based morphological profiles was introduced for classification of very high spatial resolution remote sensing images. A formal definition of OMPs and experimental evaluation were provided. OMPs are intended to allow for a very flexible and exhaustive characterization of various objects in an image with respect to morphologic operators. As such, they allow for a multi-level decomposition of an image considering also shape-related, topological, and hierarchical properties of affiliated image objects (i.e., discrete image regions). Thereby, this work can be attributed to, and extends the methodological canon of object-based image analysis techniques, which is found to represent an emerging paradigm in the context of remote sensing and geographic information science $[1]$.

The proposed technique was applied to two portions of very high spatial resolution panchromatic imagery acquired by the WorldView-II sensor. The imagery was classified according to relevant urban LULC classes within random forest architecture. Thereby, a comprehensive number of features based on OMPs was computed and employed for classification. In this, various feature sets were compiled to understand the role of the different groups of features that can be retrieved based on OMPs. The results underline the effectiveness of the proposed OMPs, which allow for obtaining a significantly increased classification accuracy of learned models compared to standard MPs, and enhanced spatial consistency of classification maps.

Subsequent works can address the extension of this concept for processing of multi- and hyperspectral imagery. Thereby, the implementation of a proper dimensionality reduction scheme (e.g., principle component analysis) appears imperative to alleviate the computational burden associated with feature selection based on filters and in particular wrapper-based methods for high-dimensional data sets. Moreover, a tailored classification approach based on e.g., a SVM with multi-source composite kernels could provide beneficial joint consideration of the spectral and spatial information. Finally, it would be very interesting to benchmark OMPs in a consistent experimental setup, which allows for stringent comparability, with respect to results obtained by other advanced mathematical morphology-based processing techniques such as attribute profiles.

\section{APPENDIX A}

Fig. 6 shows an exemplification of a $M P(I)$ and corresponding $O M P(I)$. The considered MOs opening and closing were obtained with a square-shaped SE. One segmentation level $s$ was created for the $O M P(I)$ and mode was used as aggregation function. 
(a) Morphological profile

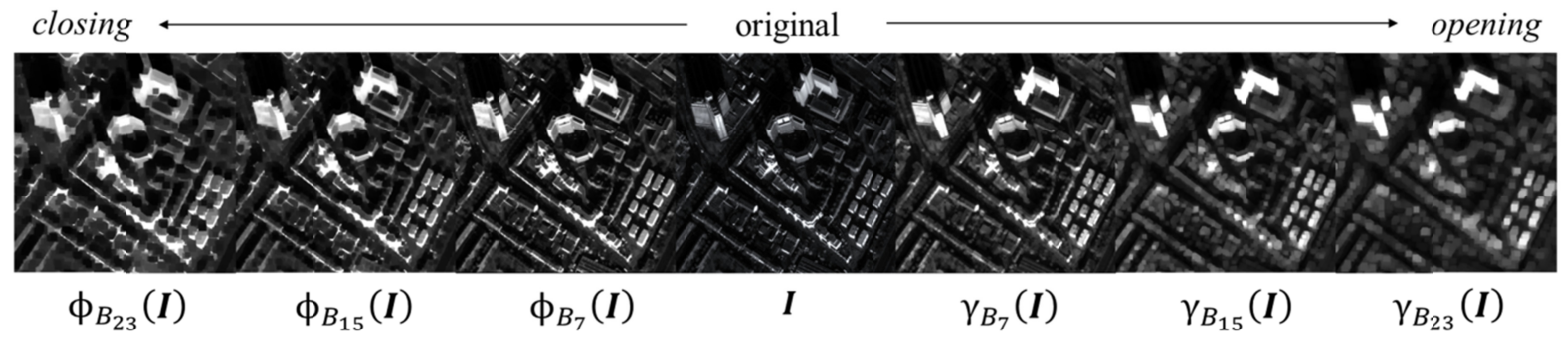

(b) Object-based morphological profile

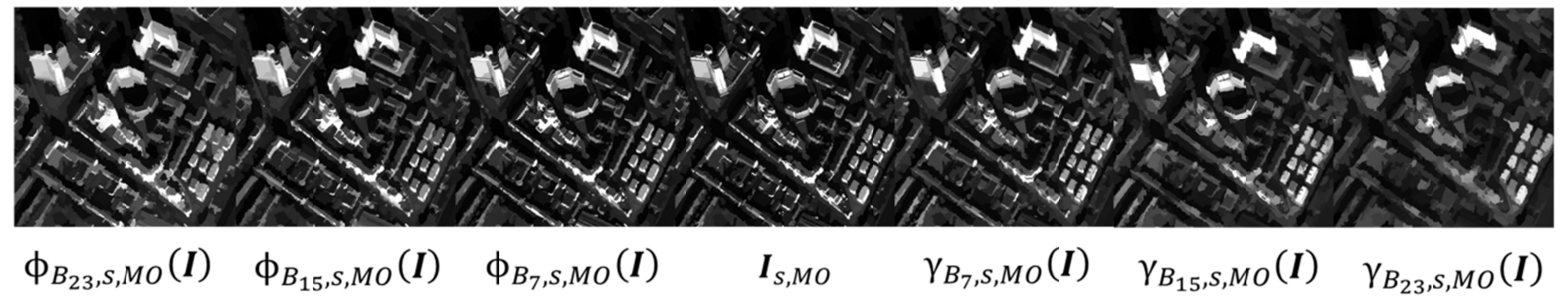

Fig. 6. Exemplification of a $M P(\boldsymbol{I})$ (a), and corresponding $O M P(\boldsymbol{I})$ (b) with respect to the MOs opening and closing.

\section{ACKNOWLEDGEMENTS}

This work was supported by the German Federal Ministry for Economic Affairs and Energy's initiative "Smart Data-innovations from data" under grant agreement: "smart data for catastrophe management (sd-kama, 01MD15008B)." The authors want to thank the four anonymous reviewers for their very helpful comments and suggestions.

\section{REFERENCES}

[1] T. Blaschke, et al., "Geographic Object-Based Image Analysis - Towards a new paradigm," ISPRS Journal of Photogrammetry and Remote Sensing, vol. 87, pp. 180191, 2014.

[2] P. Soille, Morphological Image Analysis: Principles and Applications, $2^{\text {nd }}$ ed. Berlin, Germany: Springer: 2004.

[3] D. Tuia, F. Pacifici, M. Kanevski, W. J. Emery, "Classification of Very High Spatial Resolution Imagery Using Mathematical Morphology and Support Vector Machines," IEEE Transactions on Geoscience and Remote Sensing, vol. 47, no. 11, pp. 38663879, Nov. 2009.

[4] J. Serra, Image Analysis and Mathematical Morphology. Orlando, FL, USA: Academic Press, 1983.

[5] M. Pesaresi, J. A. Benediktsson, "A New Approach for the Morphological Segmentation of High-Resolution Satellite Imagery," IEEE Transactions on Geoscience and Remote Sensing, vol. 39, no. 2, pp. 309-320, Feb. 2001.

[6] J. A. Benediktsson, M. Pesaresi, K. Arnason, "Classification and Feature Extraction for Remote Sensing Images From Urban Areas Based on Morphological 
Transformations," IEEE Transactions on Geoscience and Remote Sensing, vol. 41, no. 9, pp. 1940-1949, Sep. 2003.

[7] J. A. Benediktsson, J. Aevar Palmason, J. R. Sveinsson, "Classification of Hyperspectral Data From urban Areas Based on Extended Morphological Profiles," IEEE Transactions on Geoscience and Remote Sensing, vol. 43, no. 3, pp. 480-491, Mar. 2005.

[8] M. Fauvel, J. A. Benediktsson, J. Chanussot, J. R. Sveinsson, "Spectral and Spatial Classification of Hyperspectral Data Using SVMs and Morphological Profiles," IEEE Transactions on Geoscience and Remote Sensing, vol. 46, no. 11, Nov. 2008.

[9] M. Fauvel, J. Chanussot, J.A. Benediktsson, "A spatial-spectral kernel-based approach for the classification of remote-sensing images," Pattern Recognition, vol. 45, pp. 381$392,2012$.

[10] M. Fauvel, Y. Tarabalka, J. A. Benediktsson, J. Chanussot, J. C. Tilton, “Advances in Spectral-Spatail Classification of Hyperspectral Images," Proceedings of the IEEE, vol. 101, no. 3, Mar. 2013.

[11] X. Huang, X. Guan, J. A. Benediktsson, L. Zhang, J. Li, A. Plaza, M. Dalla Mura, "Multiple Morphological Profiles From Multicomponent-Base Images for Hyperspectral Image Classification," IEEE Journal of Selected Topics in Applied Earth Observation and Remote Sensing, vol. 7, no. 12, pp. 4653-4669, Dec. 2014.

[12] A. Daamouche, F. Melgani, N. Alajlan, N. Conci, "Swarm Optimization of Structuring Elements for VHR Image Classification," IEEE Geoscience and Remote Sensing Letters, vol. 10, no. 6, pp. 1334-1338, Nov. 2013.

[13] Z. Y. Lv, P. Zhang, J. A. Benediktsson, W. Z. Shi, "Morphological Profiles Based on Differently Shaped Structuring Elements for Classification of Images With Very High Spatial Resolution, “ IEEE Journal of Selected Topics in Applied Earth Observation and Remote Sensing, vol. 7, no. 12, pp. 4644-4652, Dec. 2014

[14] M. Dalla Mura, J. A. Benediktsson, B. Waske, L. Bruzzone, "Morphological Attribute Profiles for the Analysis of Very High Resolution Images," IEEE Transactions on Geoscience and Remote Sensing, vol. 48, no. 10, pp. 3747-3762, Oct. 2010.

[15] E. J. Breen, R. Jones, “Attribute Openings, Thinnings, and Granulometries," Computer Vision and Image Understanding, vol. 64, no. 3, pp. 377-389, Nov. 1996.

[16] M. Dalla Mura, A. Villa, J. A. Benediktsson, J. Chanussot, L. Bruzzone, "Classification of Hyperspectral Images by Using Extended Morphological Attribute Profiles and Independent Component Analysis," IEEE Geoscience and Remote Sensing Letters, vol. 8, no. 3, pp. 542-546, May 2011. 
[17] N. Falco, J. A. Benediktsson, L. Bruzzone, "Spectral and Spatial Classification of Hyperspectral Images Based on ICA and Reduced Morphological Attribute Profiles," IEEE Transactions on Geoscience and Remote Sensing, vol. 53; no. 11, pp. 62236240, Nov. 2015.

[18] N. Falco, M. Dalla Mura, F. Bovolo, J. A. Benediktsson, L. Bruzzone, "Change Detection in VHR Images Based on Morphological Attribute Profiles," IEEE Geoscience and Remote Sensing Letters, vol. 10, no. 3, pp. 636-640, May 2013.

[19] J. Li, H. Zhang, L. Zhang, "Supervised Segmentation of Very High Resolution Images by the Use of Extended Morphological Attribute Profiles and a Sparse Transform,“ IEEE Geoscience and Remote Sensing Letters, vol. 11, no. 8, pp. 1409-1413, Aug. 2014.

[20] S. Song, J. Li, M. Dalla Mura, P. Li, A. Plaza, J. M. Bioucas-Dias, J. A. Benediktsson, J. Chanussot, "Remotely Sensed Image Classification Using Sparse Representations of Morphological Attribute Profiles," IEEE Transactions on Geoscience and Remote Sensing, vol. 52, no. 8, pp. 5122-5136, Aug. 2014.

[21] P. Ghamisi, M. Dalla Mura, J. A. Benediktsson, "A Survey on Spectral-Spatial Classification Techniques Based on Attribute Profiles," IEEE Transactions on Geoscience and Remote Sensing, vol. 53, no. 5, May 2015.

[22] R. L. Kettig, D. A. Landgrebe, "Classification of Multispectral Image Data by Extraction and Classification of Homogeneous Objects," IEEE Transactions on Geoscience Electronics, vol. GE-14, no. 1, Jan. 1976.

[23] T. Blaschke, "Object based image analysis for remote sensing," ISPRS Journal of Photogrammetry and Remote Sensing, vol. 65, pp. 2-16, 2010.

[24] L. Bruzzone, L. Carlin, “A Multilevel Context-Based System for Classification of Very High Spatial Resolution Images," IEEE Transactions on Geoscience and Remote Sensing, vol. 44, no. 9, pp. 2587-2600, Sep. 2006.

[25] H. G. Akçay, S. Aksoy, "Automatic Detection of Geospatial Objects Using Multiple Hierarchical Segmentations," IEEE Transactions on Geoscience and Remote Sensing, vol. 46, no. 7, pp. 2097-2111, Jul. 2008.

[26] H. Taubenböck, T. Esch, M. Wurm, A. Roth, S. Dech, "Object-based feature extraction using high spatial resolution satellite data of urban areas, “ Journal of Spatial Science, vol. 55, no. 1, pp. 117-132, Jun. 2010.

[27] T. Kuhn, “The Structure of Scientific Revolutions,” Chicago (University Press Chicago), first published 1962. 
[28] C. Geiß, H. Taubenböck, "Normalization of TanDEM-X DSM Data in Urban Environments With Morphological Filters," IEEE Transactions on Geoscience and Remote Sensing, vol. 53, no. 8, Aug. 2015.

[29] R. M. Haralick, S. R. Sternberg, and X. Zhuang, "Image analysis using mathematical morphology," IEEE Trans. Pattern Anal. Mach. Intell., vol. PAMI-9, no. 4, pp. 532550, Jul. 1987.

[30] B. Hou, T. Huang, L. Jiao, "Spectral-Spatial Classification of Hyperspectral Data Using 3-D Morphological Profile," IEEE Geoscience and Remote Sensing Letters, vol. 12, no. 12, pp. 2364-2368, Dec. 2015.

[31] Z. Sun, H. Fang, M. Deng, A. Chen, P. Yue, L. Di, "Regular Shape Similarity Index: A Novel Index for Accurate Extraction of Regular Objects From Remote Sensing Images," IEEE Transactions on Geoscience and Remote Sensing, vol. 53, no. 7, pp. 3737-3748, Jul. 2015.

[32] M. Wurm, A. Schmitt, H. Taubenböck, "Building Types' Classification Using ShapeBased Features and Linear Discriminant Functions," IEEE Journal of Selected Topics in Applied Earth Observation and Remote Sensing, in press.

[33] eCognition Developer 9.64.0 Reference Book 9.0.3. Munich, Germany: Trimble, 2014, $489 \mathrm{p}$

[34] M. Baatz and A. Schäpe, "Multiresolution Segmentation-An Optimization Approach for High Quality Multi-Scale Image Segmentation,” ser. Angewandte Geographische Informations-Verarbeitung XII, J. Strobl, T. Blaschke, and G. Griesebner Eds. Karlsruhe, Germany: 2000, pp. 12-23.

[35] G. J. Hay, T. Blaschke, D. J. Marceau, and A. Bouchard, "A comparison of three imageobject methods for the multiscale analysis of landscape structure," ISPRS J. Photogram. Remote Sens., vol. 57, nos. 5-6, pp. 327-345, Apr. 2003.

[36] M. Neubert, H. Herold, and G. Meinel, “Assessing Image segmentation quality concepts, methods and application," in Object-Based Image Analysis-Spatial Concepts for Knowledge-Driven Remote Sensing Applications, T. Blaschke, G. Hay, and S. Lang, Eds. Berlin, Germany: Springer-Verlag, 2008, pp. 769-784.

[37] C. Geiß, H. Taubenböck, “Object-based Postclassification Relearning," IEEE Geoscience and Remote Sensing Letters, vol. 12, no. 11, pp. 2336-2340, Nov. 2015.

[38] L. Breiman, "Random Forests,” Machine Learning, vol. 45, pp. 5-32, 2001.

[39] A. Stumpf, N. Kerle, "Object-oriented mapping of landslides using random forests," Remote Sens. Environ., vol. 115, no. 10, pp. 2564-2577, 2011. 
[40] R. Genuer, J. M. Poggi, C. Tuleau, "Random forests: some methodological insights," Rapport de recherche RR-6729, INRIA, 2008.

[41] R. Kohavi, G. J. John, "Wrappers for feature subset selection," Artificial Intelligence, vol. 97, pp. 273-324.

[42] C. Kurtz, N. Passat, P. Gancarski, A Puissant, "Extraction of complex patterns from multiresolution remote sensing images: A hierarchical top-down methodology," Pattern Recognition, vol. 45, no. 2, pp. 685-706, Feb. 2012.

[43] G. M. Foody, "Thematic map comparison: Evaluating the statistical significance of differences in classification accuracy," Photogramm. Eng. Remote Sens., vol. 50, no. 5, pp. 627-633, 2004.

[44] C. Goutte and E. Gaussier, "A probabilistic interpretation of precision, recall and Fscore, with implication for evaluation," in Proc. Adv. Inf. Retrieval, 2005, vol. 3408, pp. 345-359. 